\title{
Modeling Supply Risk using Belief Networks: A Process with Application to the Distribution of Medicine
}

\author{
K. Leerojanaprapa ${ }^{1,2}$, R. van der Meer $^{2}$, L. Walls ${ }^{2}$ \\ ${ }^{1}$ King Mongkut's Institute of Technology Ladkrabang (KMITL), Bangkok, Thailand \\ ${ }^{2}$ Department of Management Science, University of Strathclyde, Glasgow, Scotland
}

(klkanogk@kmitl.ac.th)

\begin{abstract}
We propose a modeling approach based on belief networks to capture and understand the systemic nature of risks affecting supply networks. By aligning the purpose of a model with the nature of supply management decisions, we provide a mechanism for identifying relevant supply risks so that we can visualize interdependencies between risks and predict their effects on supply performance. By using a belief network modeling formalism we can use diagnostics to understand the key drivers of unwanted risk scenarios and to explore the efficacy of possible risk mitigating actions. We illustrate how belief network modeling can be used to manage the risk/reward position and provide new insights into supply risks through an example for the medicine supply chain of a regional health service provider.
\end{abstract}

Keywords - Belief network, medicine supply, supply chain, risk management

\section{INTRODUCTION}

Risk management in supply networks (SN) has been a growing area of research in operations, supply chain and management science [1]. A major challenge facing supply chain management is that many recent strategies and approaches to SN design, which aim to increase supply chain efficiency, actually tend to make the SN more vulnerable and exposed to risks. For example: lean and just-in-time strategies seek to eliminate resource wastage but may end up reducing buffers against supply risk; outsourcing and increased reliance on global transport and logistics are meant to cut production costs but may introduce new sources of SN risk. Consequently we need to better understand and proactively manage $\mathrm{SN}$ risk.

To date, SN risk research has focused upon understanding and classifying the source. Such classification taxonomies implicitly assume that risks can be categorized into mutually exclusive classes. However, it is acknowledged that risks in increasingly complex SN are interconnected [2], [3]. Therefore classifications can be useful in typifying sources of risk but are not sufficient for effective management. In this article, we argue that modeling provides a means of capturing and analyzing the inter-dependencies between risks to support useful decisions about SN management.

As an example, consider our motivating problem which relates to a medicine supply in NHS Greater Glasgow and Clyde (NHS GG\&C). This is the largest
NHS organization in Scotland, serving a population of 1.2 million people. In 2010 there was a major change to the medicine supply chain. In the new medicine SN, numerous local stockholdings have been replaced by a large, robot-controlled Distribution Centre (DC), which stocks medicines from multiple external suppliers and distributes medicines to all hospital wards and pharmacies within the NHS GG\&C area. The intended benefits included: (a) increased efficiency based on the riskpooling effect of centralized inventory holding in the presence of stochastic demands for medicines; and (b) improved customer service through the high order-picking speeds and accuracy of the distribution robots.

As with any project designed to implement a new process, risk management was critical: "The overall goal of risk management is to have an environment of "No Surprises' where we understand the risks we face and eliminate or control them to an acceptable level, by creating a culture founded upon assessment and prevention of risk." [4, p. 5]. NHS GG\&C used risk registers, a standard risk management approach. A review of the implementation of the new medicine SN acknowledged that many of the anticipated risk drivers were in fact more complex than initially believed because of the inter-dependencies between risks that had the potential to impact supply. For example, risks such as changes in staff morale or a new IT system were anticipated but appeared as independent events in the risk register. In reality these risks were systemic because of adverse interactions between IT and robotics problems with attitudes of, and actions by, staff relating to the new technology.

In this article we discuss how modeling can support identification of $\mathrm{SN}$ risk events and their interrelationships. We adopt Belief Networks (BN) [5] as our modeling formalism for the following reasons: a BN can represent uncertain relationships between risk events; the uncertainty regarding the occurrence of a risk event can be quantified as a probability; and analysis grounded in the theory of Bayesian methods can be conducted. BN have been used in other risk management applications, but apart from [6], [7] there has been little consideration of their use in a SN context.

Section II provides an overview of our general BN modeling approach for SN risk. In Section III we present a selection of analysis of the SN risk modeling of the NHS GG\&C medicine supply to hospital inpatients. We summarize our conclusions and reflections in Section IV. 


\section{BN MODELING PROCESS FOR SN RISK}

\section{A. Belief Network Modeling Concept}

Fig. 1 shows the key concepts underpinning our approach to $\mathrm{BN}$ modeling for $\mathrm{SN}$ risk. The diagram represents the cause-effect relations between SN risk events. The "effect" corresponds to the SN performance measure of interest. The "causes" may include both root causes as well as intermediate events. Risk mitigation strategies may be selected to impact "causes" or "effects".

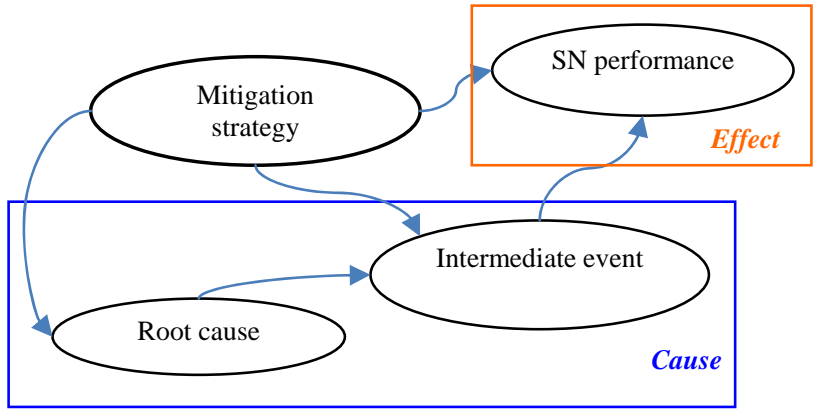

Fig. 1. Belief network (BN) SN risk modeling concept

The ultimate "effect" may correspond to overall SN performance. For example, costs, quality, or customer service level performance depending on type of SN and the organizational goals. The "causes" of SN risk events are extensive but we assume these can be due to disruptions caused by the external environment, demandside uncertainty, supply-side uncertainty and vulnerabilities internal to the organization [8]. Uncertainty in the occurrence of the "effect" events are expressed as a probability conditional on their "causal" events.

A risk mitigation strategy will encompass a set of actions designed to remove or reduce the effect of an event. For example, by removing the root cause, or providing a barrier to reduce an intermediate event threat, or providing an alternative supply route through back-up provision. Note, however, that the usual risk mitigation tactics of redundancy and diversity may fundamentally alter the risk/reward position of the organization.

\section{B. Key Stages of Belief Network Modeling}

Our model building process, summarized in Table I, is informed by general approaches for BN modeling [9] and is grounded in our experience of risk analysis [10].

We assume a meta-stakeholder exists within the client organization with ownership of the SN and a commitment to support risk analysis. Such a person plays important gatekeeping and championing roles thus ensuring modeling is aligned with supply risk management.

Building real $\mathrm{BN}$ is both an art and a science since the goal is to construct a model that appropriately represents stakeholder knowledge. While the main stages in our scientific model building, as explained in the remainder of this Section, may appear very structured, it is important to note that in practice these stages are not necessarily linear and iteration between activities is common.
TABLE I

PROCESS TO DEVELOP BELIEF NETWORK SN RISK MODEL

\begin{tabular}{|c|c|c|c|}
\hline Stage & Activity & Method & Participants \\
\hline 1 & $\begin{array}{l}\text { Identify SN scope } \\
\text { and stakeholders }\end{array}$ & Interview & Meta-stakeholder \\
\hline 2 & $\begin{array}{l}\text { Visual } \\
\text { representation of } \\
\text { SN process flow }\end{array}$ & $\begin{array}{l}\text { Documentation } \\
\text { Interview }\end{array}$ & Stakeholders \\
\hline 3 & $\begin{array}{l}\text { Gain qualitative } \\
\text { understanding of } \\
\text { SN risk events }\end{array}$ & Interview & $\begin{array}{l}\text { Stakeholders, } \\
\text { Meta-stakeholder }\end{array}$ \\
\hline 4 & $\begin{array}{l}\text { Structure the } \\
\text { provisional BN SN } \\
\text { risk model }\end{array}$ & $\begin{array}{l}\text { Align with BN } \\
\text { formalism }\end{array}$ & Modeler \\
\hline 5 & $\begin{array}{l}\text { Refine the BN SN } \\
\text { risk structure }\end{array}$ & Workshop & $\begin{array}{l}\text { Stakeholders, } \\
\text { Meta-stakeholder }\end{array}$ \\
\hline 6 & $\begin{array}{l}\text { Quantify the BN } \\
\text { SN risk model }\end{array}$ & Interview & Stakeholders \\
\hline 7 & $\begin{array}{l}\text { Use BN to support } \\
\text { SN risk analysis }\end{array}$ & $\begin{array}{l}\text { Analysis with } \\
\text { software tool }\end{array}$ & Modeler \\
\hline 8 & $\begin{array}{l}\text { Validate BN SN } \\
\text { risk model behavior }\end{array}$ & Workshop & $\begin{array}{l}\text { Stakeholder, } \\
\text { Meta-stakeholder }\end{array}$ \\
\hline
\end{tabular}

Stage 1: Identify SN scope and stakeholders Together with the meta-stakeholder we explore the main aspects of SN performance to be understood from a risk perspective. In this way we scope our "top event" or set of "top events". Using these events as a focal point we bound the SN so that we can determine relevant stakeholders, individuals or organizational units, who might contribute to modeling.

Stage 2: Visual representation of $S N$ process flow Understanding the details of the supply flows through process mapping or some other means of visualizing the $\mathrm{SN}$ as a system in an appropriate diagram, is important to support systematic investigation of potential risk events.

Stage 3: Gain qualitative understanding of SN risk events - By interviewing relevant individual stakeholders with operational experience and using the process flow as a guide, we develop causal maps to capture their causeeffect reasoning about SN risk events. The modeler, together with meta-stakeholder, will integrate the individual causal maps to generate an initial qualitative model of the SN. Workshops, or other equivalent means, can be used to bring stakeholders together to challenge the overall map and refine it through facilitated discussion.

Stage 4: Structure the provisional BN SN risk model The modeler should review the causal map representing stakeholder understanding and ensure that it conforms to the formalism of $\mathrm{BN}$. That is, structuring the $\mathrm{BN}$ so that the property of conditional independence is satisfied and that there are no cyclical relationships. Each risk event should be represented as a variable within the BN and the states of each variable, assuming they can be defined as discrete states (e.g. bad snow, light snow, no snow) or they can be discretized (e.g. material flow). For more information on the BN modeling formalism, see [5] and for guidance on translating causal maps to a $\mathrm{BN}$ see [11].

Stage 5: Refine the BN SN risk structure - A facilitated workshop with stakeholders and Meta- 
stakeholder allows review of the BN structure and the definition of model variables. The aim is to challenge the $\mathrm{BN}$ and refine it so that there is agreement on the representation of the risk events to be included in the model as well as ensuring the anticipated dependencies between risks have been captured appropriately.

Stage 6: Quantify the BN SN risk model - Prior to interviewing individual stakeholders, a questionnaire can be developed to frame questions used to obtain subjective probability assessments of the chance of occurrence of risk events. During the interview such questions might be asked about the likely frequency of events. For example, "Think about urgent orders to the DC (during May 2012 to April 2013). In 100 medicines which could be ordered urgently for the DC, how many of them could be requested but might not be in the DC stock catalogue?".

Stage 7: Use BN to support SN risk analysis - Several software tools exist to support BN analysis. We have used GeNIe [12]. Typically, each tool has common basic functions for analysis of, for example, the chance of the top event (i.e. performance of $\mathrm{SN}$ given the risk events to which it is exposed). Or, to control particular states of that top event, or indeed any other event, to identify which are the main causes that would most likely lead to that state of the event occurring. The former analysis allows SN performance to be predicted under risk and uncertainty for particular situations, while the latter analysis allows causes of unwelcome events to be prioritized for action.

Stage 8: Validate BN SN risk model behavior - Face validity can be achieved by gaining feedback from stakeholders on model structure and resulting outcomes. We can establish the degree of sensitivity of the BN to changes in variables or the probabilities associated with their states. Scenario analysis can be used to explore meaningful cases defined by stakeholders to investigate the behavior of the SN under possible risk futures.

\section{ANALYSIS OF MEDICINE SUPPLY RISK}

We present example analysis of the medicine supply risk in NHS GG\&C. Our analysis was conducted after the initial project to introduce the new medicine supply was embedded within operations and was being reviewed. We focus here on the risks associated with the ongoing dayto-day process of medicine supply within the new SN, and not on the risks that had been associated with the prior project to implement the new SN design. We explain the key characteristics of the medicine supply chain, discuss how the modeling process as in Table I was implemented, then present selected analysis.

\section{A. NHS GG\&C Medicine $S N$}

Fig. 2 provides a simplified schematic of the hospital medicine SN showing the agents on the supply and demand side of the DC, our focal point. The 14 hospitals in the NHS GG\&C area represent the agents on the demand side of the DC. Each hospital has a single dispensary/pharmacy as well as multiple ward cupboards (the quantity of which depends on the size of hospital).
An innovation has been to connect inpatients directly to the SN by asking inpatients to bring their personal medicines when admitted to hospital. This is termed Patients Own Drugs (POD). Appropriate quality checks are made before storage of a patient's own medicines in his/her bedside POD locker. The medicines in each patient's locker can then be topped up from either the cupboard on the patient's ward or the hospital dispensary. When patients are discharged, they will normally take the remaining medicines in their locker home with them.

The main medicines required for any specific treatment will be prepared and stocked in the ward to which the patient is admitted. If an inpatient needs medicines that are not available from the ward stock (e.g. either not being listed in the ward's stock catalogue or out of stock at the time) then staff can use the Individual Patient Supply (IPS) system to order the medicine as an urgent request, providing a back-up process. The hospital dispensary will be responsible for filling this request. If the medicine is not available in the hospital dispensary, dispensary staff can follow a standard process in order to source the medicine from other wards in the hospital, other local hospitals, DC, other suppliers, or other hospitals, in that order of priority. All medicines for a particular inpatient will be stored in a personal POD locker that is located the patient's bedside.

On the supply side, all general medicines can be ordered by and delivered to the DC, primarily from wholesalers and manufacturers.

NHS GG\&C aims to have an agile medicine SN, which is achieved by having sufficiently high flexibility to absorb varying inpatient requirements. The DC is meant to provide regular top-ups for hospital dispensaries and ward cupboards, while the IPS system functions as a backup system to respond to urgent needs from individual inpatients. However, while flexibility and agility are highly important, cost considerations cannot be neglected by any means because of the very high medicines expenditure by the organization (about $£ 120$ million per year). In other words, NHS GG\&C must closely monitor its risk/reward position.

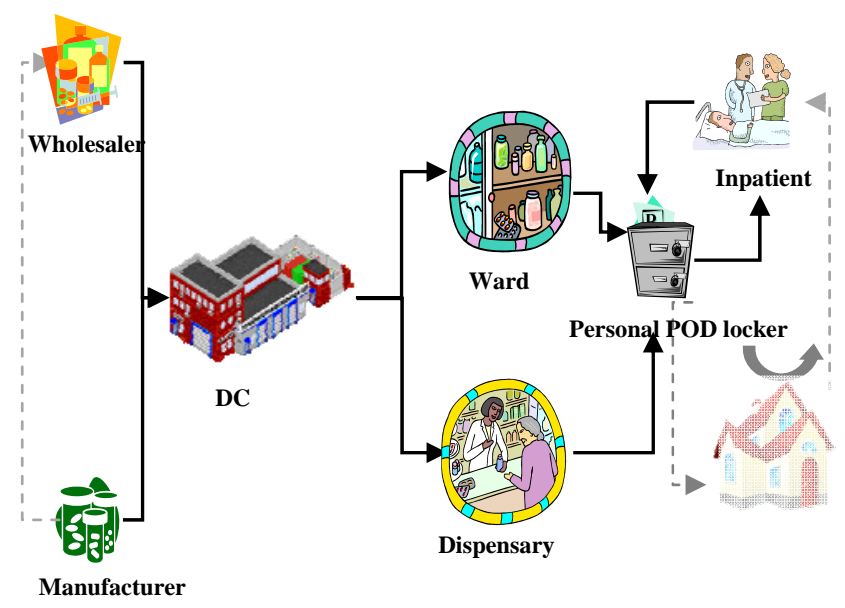

Fig. 2. Simplified schematic of NHS GGC\&C hospital medicine supply 
Our analysis considers a SN with four types of organizational unit - the DC, external suppliers, hospital dispensary (one per hospital), and hospital wards. Eight stakeholders from these units, including a procurement manager, pharmacists, and distribution technician, have been involved in expressing their beliefs about the nature and likelihood of medicine supply risks. The Lead Pharmacist for NHS GG\&C assumed the role of a metastakeholder to constantly support and challenge modeling. The case was conducted between March-December 2012.

\section{B. Medicine Supply BN Model}

Fig. 3 shows a partial extract from the BN model. The overall model contains 66 nodes, representing risk events, and 69 arcs, capturing relationships between these events.

The top event is defined as a shortfall in performance. That is, as a "failure to supply the right medicine to the right patient at the right time". The extract from the BN model in Fig. 3 shows one cause of this overall SN failure occurs when medicine is not available in the POD locker. In turn this event might be due to multiple causes.

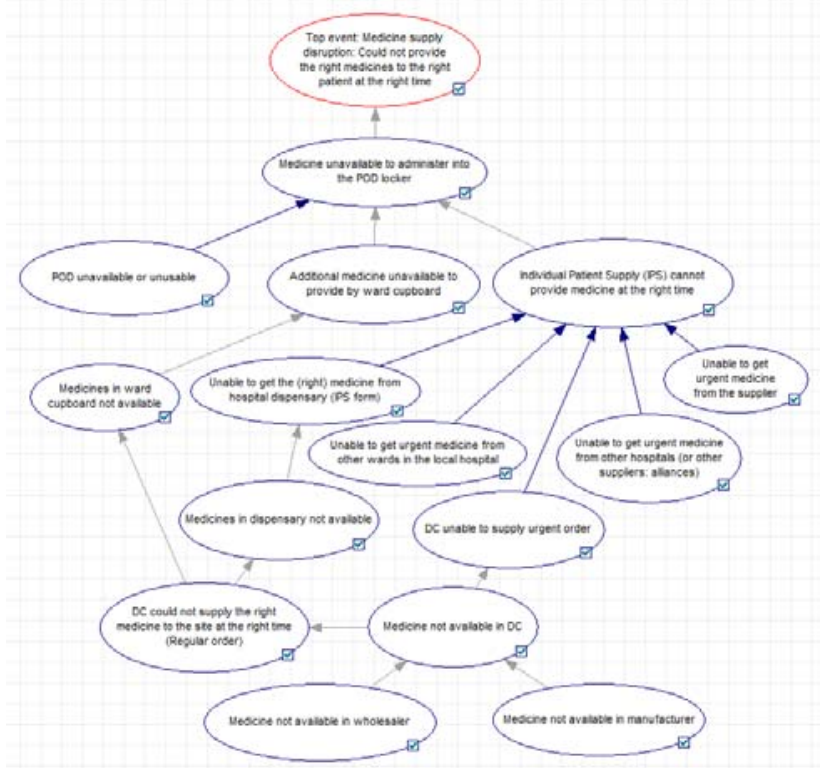

Fig. 3. Partial extract from the medicine supply BN model

\section{Selected Findings from Medicine Supply Analysis}

We now discuss extracts from our analysis. First, we investigate scenarios that could directly affect chance that the right medicine is not available at the right time to be put into the bedside (POD) locker of a specific hospital inpatient. What are the possible causes for this?

Medicines are available from at least five alternative sources, including: (1) the patient's own medicines taken into the hospital upon admission; or (2) the stock of medicines normally kept on the ward (according to the specific stock list for that ward); or (3) the stock of medicines in the hospital dispensary (supplying all wards in the hospital); or (4) the medicines stored in the DC; or (5) medicines sourced from other wards in the hospital, or from other hospitals nearby, or directly from a wholesaler.
Any of these five sources might cause a failure to deliver at any time, but for "the right medicine not to be available at all”, all potential medicine sources would have to fail to deliver on demand. This redundancy of supply represents a deliberate design feature in the SN to keep the risk of non-supply as low as practically possible.

Beliefs about the chance of medicine being unavailable from each source have been elicited from the stakeholders in the form of subjective probabilities. Populating the $\mathrm{BN}$ with these probabilities allows us to estimate the chances of the top event, which we assume is in one of the three states: unavailable; unsuitable quality; both unavailable and unsuitable quality. Unavailable means that a medicine is not available at all. Unsuitable captures situations where, for example, a medicine is beyond its expiry date or is not available in the correct dosage. If a patient has been prescribed a set of medicines in combination, then it could be possible for one of these medicines to be unavailable and for another to be available but not of a suitable quality.

For the operational medicine SN, the model estimates that the chance of the right medicine not being available for the right patient at the right time is approximately five times that of the chance of the medicine being unsuitable. The chance of one medicine on a particular patient's prescription being unavailable and another medicine on that same prescription being unsuitable is negligible.

We now examine scenarios corresponding to the lack of a specific medicine at one or more of the three main storage locations. We set the probabilities of selected "effect" variables, which represent a scenario where medicine is not available from a given source, so that we may re-estimate the overall probability of the top event. The scenario analysis results are summarized in Fig. 4. Note that the numerical values are deliberately not shown.

The bar chart shown in Fig. 4 indicates that medicine unavailability in either the hospital ward, or the hospital dispensary, or the DC, has a relatively small impact on the chance of the right medicine not being available to a specific inpatient compared with situations where there is unavailability at multiple sources. As we might expect, the chance of failing to provide medicine to a patient is highest when a required medicine is neither present in the ward cupboard, nor in the hospital dispensary, nor in the DC. Such a risky event combination might occur under circumstances such as patient admissions outside normal working hours (i.e. on weekends/night/ holiday periods).

As we might expect, we find that that probabilities estimated under our scenario analysis always exceed those for the actual medicine supply. For example, under the scenario that medicine is simultaneously unavailable from the ward and the hospital dispensary, the probability of the top event increases by $28 \%$ above that estimated for the actual medicine SN. When, in addition, the medicine is unavailable from the regional DC as well as the hospital dispensary and the ward, then the probability increases by $132 \%$ over the estimated value for the actual medicine supply. 


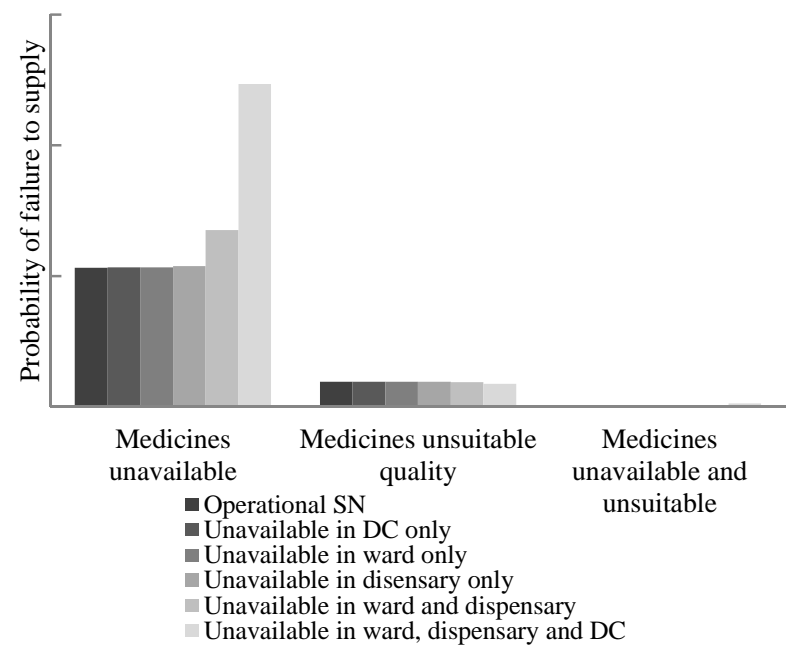

Fig. 4. Relative chances of being able to supply the right medicines to the right patient at the right time under 5 source unavailability scenarios

\section{REFLECTIONS AND CONCLUSIONS}

In our example, various back-up medicine supply systems exist. Therefore, the medicine SN is normally highly secure and a high level of responsiveness to inpatients' medicine requirements can be maintained. Management attention needs to be directed at those specific situations, such as out-of-hours admissions, where a combination of adverse events is more likely to occur. Rather than maintaining excess medicine stocks at ward level or dispensary level to provide some general level of protection, as has tended to be the case in practice, the risk/reward position should be moved in a more favorable direction by focusing on particular highrisk situations. For instance, by creating a backup source of supply that specifically targets those situations. Our example shows the benefit of looking at scenarios combining various adverse events, which cannot be easily done with conventional risk register.

Our analysis indicates that participating in the development of a BN SN risk model of the kind discussed in this article can help managers and other staff involved in supply chain operations to solve problems more effectively. For example, one of our stakeholders noted that modeling the supply process and its associated risks helped them to identify problems with particular combinations of adverse events more effectively and more easily. Our evaluation feedback suggests that the workshop discussions enabled stakeholders to develop a shared understanding of the possible impact of adverse events. Some events that they thought highly significant turned out to be less so in practice. For example, the manager of the regional DC had been much concerned by the high probability of staff shortages in her area. However, during the process of quantification, she had to provide the effect of 'shortage staff' on 'DC supply delay' and found that it did not generate the huge impact that she had previously thought. This was just one of benefits from a modeling exercise, as it helped this manager to prioritize the key risks rather than trying to cover every possible angle. More generally, the modeling exercise uncovered opportunities for focusing on particular high-risk combinations of events, rather than employing excess resources in circumstances where that was less warranted. This could help to move the organization's risk/reward position in a more favorable direction.

\section{ACKNOWLEDGMENTS}

We would like to thank to all the staff of NHS GG\&C who participated in model building. We acknowledge the sponsorship from the Royal Thai Government and King Mongkut's Institute of Technology Ladkrabang (KMITL), Thailand, for Kanogkan Leerojanaprapa’s PhD study.

\section{REFERENCES}

[1] M. S. Sodhi and C. S. Tang, Managing Supply Chain Risk. London: Springer, 2012.

[2] S. Chopra and M. S. Sodhi, "Managing risk to avoid supply-chain breakdown," Mit Sloan Management Review, vol. 46, no. 1, pp. 53-61, 2004.

[3] B. Ritchie and C. Brindley, "Supply chain risk management and performance; A guiding framework for future development," Int. J. Operations \& Production Management, vol. 27, no. 3, pp. 303-322, 2007.

[4] NHS Greater Glasgow \& Clyde, "Risk Management Strategy,” 2007. [Online]. Available:

http://library.nhsggc.org.uk/mediaAssets/library/nhsggc_ris k_management_strategy_2007-05.pdf. [Accessed: 22-Nov2012].

[5] U. B. Kjaerulff and A. L. Madsen, Bayesian networks and influence diagrams : a guide to construction and analysis . New York: Springer, 2008.

[6] A. A. Mouhamad Shaker, R. Van Der Meer, and L. Walls, "Modelling supply network reliability using dynamic Bayesian networks," in Hamburg International Conference on Logistics, 2012.

[7] G. Basu, M. Ben-Hamida, K. Butner, E. Cope, H. Dao, L. Deleris, J. Dong, M. Helander, K. Katricioglu, B. Ray, and J. Torpy, "Supply chain risk management: A delicate balance act A multi-faced view on managing risk in a global integrated enterprise," IBM business consulting services, New York, 2008.

[8] U. Jüttner, "Supply chain risk management understanding the business requirements from a practitioner perspective," The International Journal of Logistics Management, vol. 16, no. 1, pp. 120-141, 2005.

[9] J. H. Sigurdsson, L. Walls, and J. Quigley, "Bayesian belief nets for managing expert judgement and modelling reliability," Quality and Reliability Engineering International, vol. 17, pp. 181-190, 2001.

[10] K. Leerojanaprapa, L. Walls, and R. Van der Meer, "Modelling and managing systemic risks in supply chains," in 18th Int Annual EurOMA Conference, 2011, p. 112.

[11] S. Nadkarni and P. P. Shenoy, "A Bayesian network approach to making inferences in causal maps,” Euro. J. of Operational Research, vol. 128, pp. 479-498, 2001.

[12] The Decision Systems Laboratory, "GeNIe and SMILE," 2013. [Online]. http://genie.sis.pitt.edu/about.html. [Accessed: 06-Jan-2013]. 